COMMUNICATIONS

\title{
DEGENERATIONS OF THE DOG RETINA*
}

\section{STRUCTURE AND DEVELOPMENT OF THE RETINA OF THE NORMAL DOG}

BY

\author{
H. B. PARRY \\ Animal Health Trust, Kennett, Newmarket, Suffolk
}

DURING the course of an investigation to define and control an hereditary form of generalized progressive retinal atrophy (hereditary degeneration of the retina) in dogs of the Red Irish Setter breed (Hodgman, Parry, Rasbridge and Steel, 1949; Parry, 1951), certain other syndromes of retinal degeneration were encountered. In an attempt to define these syndromes clinically and histologically it became necessary to review our knowledge of the structure of the retina of the normal dog and its variation with breed and age.

Johnson (1901) recorded ophthalmoscopic observations on the fundi of Canidae amongst the many different genera he investigated, and Gray (see Nicolas, 1925) has recorded clinical observations on the dog. Detailed studies of the morphology of the dog retina go back to the classical papers of Schultze (1866) and Ramon y Cajal (1894), who referred to the dog inter alia, and Zürn (1902) examined the retinae of various breeds hunting by sound and by sight. In view of differences of histological technique, it seemed desirable to extend these observations and to correlate them with additional clinical and electro-physiological data.

\section{Material and Methods}

Animals.-The dogs were forty Red Irish Setters, twenty-two Greyhounds, five Border Collies, three Fox Terriers, a Foxhound, a Yellow Labrador Retriever, an Airedale, a Bulldog, a Poodle, and four Mongrels. They were studied clinically and at autopsy. Their ages varied from birth to 6 years. The age of the sucking puppies, i.e. until they are weaned approximately 6 weeks after birth or about 105 days after conception (gestation is about 63 days), is given in days after conception (post-conception age); thereafter the age was calculated as from birth (post-natal age). 32 pups from six litters of various breeds were examined fully.

Management.-The dogs were housed in isolated kennels at the research station under close supervision. The majority were born and reared in the kennels. They were fed a mash of cooked whalemeat and horse flesh which was used to steep a finely ground cereal mixture comprising oats 8 parts, wheat 2 parts, barley 2 parts, dredge corn, containing peas, beans and vetches, 1 part, or lucerne meal 1 part, to which small quantities of blood fibrinogen, whey concentrate, and bone meal were added. Cod liver oil of tested vitamin A and D potency was fed to the young pups and brood bitches.

\footnotetext{
* Received for publication March 6, 1953.
} 
The brood bitches in oestrus were kept in isolated runs to reduce as far as possible the chances of promiscuous matings; they were mated on two occasions at an interval of $48 \mathrm{hrs}$. The post-conception age of the pups was calculated from the date of the first mating.

A careful watch was kept for evidence of the common virus infections. Carré's or Laidlaw-Dunkin distemper, now termed distemper-complex (Mansi, 1951), was not present, but canine virus hepatitis was endemic in the kennels (Parry and Larin, 1951). As far as we know at present, this infection, which is widespread as a clinically inapparent infection in dogs in Western Europe, is not responsible for retinal disorder.

Clinical Examination of the Eye.-The dogs were examined with a Lister-Morton ophthalmoscope in a darkened room, usually without mydriatic medication. The pupil diameter was measured with a ruler when maximum constriction to the focused ophthalmoscope light was obtained; it varied rather widely with the direction of the light beam, but in most animals it was greatest when light fell on or about the area centralis. The reading, recorded as the ratio in millimetres of the diameter of the pupil to the outer diameter of the iris, is termed the average pupil size.

The light-dark and dark-light pupillary reactions were recorded as brisk $(++)$, sluggish $(+)$ and absent $(0)$. The response of eyelid closure to the approach of some potentially dangerous object (the eye preservation reflex) was tested by moving a hand towards the eye in such a manner that as little air movement as possible was created and without touching the long eyelashes. The lens and media were examined with a finely focused light beam and a $+12 \mathrm{D}$ lens; the fundus was usually in focus with $a-1$ to $-3 \mathrm{D}$ lens.

It was thought that immobilization of the dog under light anaesthesia might be an advantage. Some animals were anaesthetized lightly with ether, nitrousoxygen, or trilene; ether proved the most satisfactory agent. However, it was noted that with the onset of Stage 3 ether anaesthesia, the calibre of the blood vessels of the optic papilla increased very markedly, so that a papilla which was originally a pale pink in colour would become brick red. As the colour of the papilla is of some importance as a diagnostic criterion, the development of this vasodilatation, as well as the interference with the pupillary light reflexes, rendered examination of the fundus under ether anaesthesia unsatisfactory.

Vision Tests.-These were carried out by day and night in observation runs with numerous obstacles in positions with which the dogs were not familiar. It was found to be important that the obstacles should be free of rattles and odours which would serve to identify their position, for a dog with defective vision comes to rely very much on hearing and smell. The degree of vision defect was assessed roughly from 0 to 5 , representing the range from normal vision to complete blindness. This assessment was written as $0 / 5$ to $5 / 5$, a completely dayblind dog being said to have a day vision defect of $5 / 5$. Where night vision was tested, the night vision defect was expressed in a similar way.

Electroretinography.-In some of the dogs, recordings of the electroretinogram were made by a team in collaboration as described previously (Parry, Tansley, and Thomson, 1951; 1953). Wherever possible this was done shortly before death, usually between 4 and $16 \mathrm{hrs}$, occasionally up to 5 days, so that the clinical and histological findings might be correlated with the electrophysiological data. 
Autopsy and Technique of Intra-vital Fixation.-The majority of the dogs were killed under deep pentobarbital sodium anaesthesia in daylight. The two carotid arteries were canulated cranialwards and both jugular veins severed. Physiological saline or Ringer's solution, with a small quantity of sodium nitrite added (occasionally $2 \cdot 5$ per cent. gum acacia and heparin have also been added) and warmed to about $35-37^{\circ} \mathrm{C}$., was pumped gently with the aid of a light rubber bellows through the head until the blood from the jugular veins was obviously diluted; this usually took 1-2 minutes and required 50 to $250 \mathrm{ml}$. solution, approximately 10-20 ml. kg. bodyweight. The saline solution was then discontinued and the fixative similarly warmed was pumped through until it flowed freely from the jugular veins. The colour changes of the base of the tongue were a good guide to the penetration of the fixative. The animal died immediately; the eyeballs were removed at once and placed in the fixative. In a few eyes removed some hours after death from natural causes and with reduced intra-ocular tension, a small amount of fixative, usually 0.2 to $0.5 \mathrm{ml}$., was injected through a fine needle inserted into the vitreous body, either at the corneal-scleral junction or through the optic nerve head. The brain and optic nerves were dissected out carefully and examined closely for macroscopic abnormalities. The optic nerves were examined particularly for signs of hypoplasia, either congenital (Saunders, 1952) or associated with stenosis of the optic canals (Mellanby, 1943). None was observed in any dog. The brain, after further fixation in 10 per cent. neutral formol saline for a week or longer, was examined macroscopically after being cut in ten standard coronal planes. No macroscopic lesion of the optic pathway or visual cortex was observed. In a few cases the brains were also examined histologically to establish the absence of significant microscopic lesions.

Histology.-The fixatives used were 10 per cent. neutral formol saline

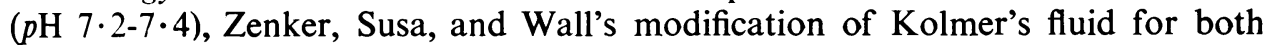
warm-blooded and cold-blooded animals (Walls, 1938). The last gave by far the most satisfactory preparations, particularly after intravital fixation, and we adopted it as our standard method. Formol saline did not give good cytological detail. Fixation after death was always associated with pyknosis of nuclei and other changes not observed in properly fixed preparations.

After fixing for one week, the eyeballs were usually cut open vertically (a piece of cotton was inserted at the mid-dorsal point at autopsy) using a sharp safety razor blade applied first at the optic nerve head. Occasionally eyes were cut horizontally and at various intermediate positions. The eyes were examined for macroscopic abnormalities, the lenses removed, and the half-eyes embedded. In a small series the portions containing the area centralis were cut serially at $20 \mu$.

The early material was embedded in gelatin and cut frozen after the method of Oakley (1937), but this gave unsatisfactory preparations and was discontinued. The later material was embedded mostly in paraffin, occasionally in celloidin, and cut at 5 to $8 \mu$. The sections were stained by Weigert's haematoxylin and eosin (after Kolmer's fixatives the nuclei do not stain well with Ehrlich's haematoxylin), Heidenhain's iron haematoxylin counterstained with aniline blue and orange G., Mallory's phosphotungstic acid, the azo-carmine (azan), and Feulgen's methods. In addition a small number of sections were stained by Weil's, Nissl's methylene blue, Holmes' silver impregnation, and the gallocyanin (Einarson, 1951) methods. The following accounts are based on intravitally fixed material using Wall's modification of Kolmer's cold-blooded fixative and paraffin embedding. The 
measurements of these sections, although given in $\mu$, are only intended as an approximate measurement to give a guide to the relative sizes, since the changes due to shrinkage and distortion during fixation are not known accurately (e.g. the diameters of erythrocytes in the blood vessels of this material were only $4 \cdot 5-5 \cdot 0 \mu$ instead of the normal $6 \cdot 5-7 \cdot 0 \mu$ of fresh dog erythrocytes in air-dried films). The anatomical terminology follows Polyak (1941).

Criteria of Normality.-The medical histories of all dogs were carefully kept. Only dogs without clinical evidence of eye lesions or defects of vision were used. Wherever possible the electroretinogram (ERG) was taken and only animals with normal $b$ - and slow secondary waves were regarded as normal (Parry, Tansley and Thomson, 1951). If there was the possibility of the dog's having been exposed to distemper, the animal was examined for any neurological defects suggesting that the infection had affected the nervous system. Similarly, careful attention was paid to the diet to rule out the nutritional disturbance of vision associated with inadequate vitamin $\mathrm{A}$ intake, which may affect the rods and cones (Tansley, 1933b; Johnson, 1939, 1943; Andersen and Hart, 1943), or cause stenosis of the optic canal (Moore and others, 1935; Hart and Guilbert, 1937), a syndrome reported in the dog by Mellanby (1943).

\section{Results}

\section{(1) Fundus And Retina - OF the Adult Dog}

(a) Clinical Data.-The fundus of the dog differs from that of man in the presence of a tapetum lucidum and a heavily pigmented choroid, as well as in the absence of a macula or fovea. It can be divided conveniently into two portions: the tapetal fundus in the dorsal quadrants backed by the brightly reflecting tapetum lucidum, and the non-tapetal fundus dark with choroidal pigment in the ventral quadrants. The tapetal fundus is half-moon-shaped, occupying most of the dorsal quadrants, but not extending to the periphery of the fundus, where it is surrounded by a narrow zone of non-tapetal fundus approximately $1 / 20$ th of the diameter of the eye-ball.

The colour of the tapetal reflection by direct retinoscopy varies from a light lemon yellow through orange to a deep apple green, in many dogs the different colours producing an effect not unlike a painting by Monet. The greyhounds and terriers have a predominantly yellow-green reflection, while the Irish Setters have, in addition, a conspicuous orange component, which in certain families is a deep orange-red. In some breeds, such as pointers, the colour of the reflection appears to be inherited with certain coat colours (e.g., liver coat colour and a light orange brown tapetum), but in most breeds this association is not very distinct, although in one tricolour bulldog the tapetal reflection was a light blue-grey with violet towards the periphery of the tapetum.

Over the tapetal fundus, the retina appears to be fully translucent without refractive distortion and its surface is flat, but the substance of the fundus appears to have a definite texture of fine granular beading, which becomes slightly coarser towards the periphery. Between the beading is a very fine 
reticulum of dark triangular spaces, the stellullae of Winslow. The granular beading is most marked in adolescent dogs; in some animals over 2 years old the texture of the granular beading is so fine that it appears to be even. A single group of primary dorsal retinal blood vessels arises from the central retinal artery and vein and passes dorsally from the disc, where it gives rise to numerous secondary branches which ramify upwards and outwards as sharply defined thin-walled vessels lying on or just within the surface layers of the retina. No macula was observed; the area centralis is only detectable by a slight reduction in the density of the secondary blood vessels over a portion of the tapetal fundus, dorsal and temporal to the disc. At the periphery of the tapetal fundus the tapetum becomes irregular with areas of dark non-tapetal fundus interposed; occasionally similar areas free of tapetum occur in the mid-tapetal fundus.

The non-tapetal fundus is a deep nigger-brown colour, due to the choroidal pigment, with a slight grey over-wash. The granular texture is not so well marked in this region. The blood vessels arising from the two main medial and lateral horizontal retinal vessels are more numerous over the ventral nontapetal fundus than over the tapetal fundus and by direct retinoscopy the larger vessels reflect the light from their surface, producing a thin regular "silver wiring" reflection, which is not seen with the tapetal vessels.

The optic papilla usually lies just within the ventral border of the tapetal fundus not infrequently surrounded by a narrow zone of non-tapetal fundus; it is round or slightly oval ; its margins are ordinarily fairly well demarcated, although in some animals they are quite ragged. There are three main groups of blood vessels, the dorsal and the two horizontal, medial and lateral. The venous vessels usually arise from a circular vessel connected to the central retinal vein while the three arterial vessels arise independently in the substance of the papilla near its centre. At the junction of the peripheral and central portions of the papilla some ten to fifteen small subsidiary arterial vessels arise within the substance of the papilla and run radially outwards over the peripapillary retina. These vessels, which are similar in disposition and calibre to the cilio-retinal vessels of the horse and were seen in sections to arise from choroidal vessels, appear to be responsible for much of the pink colour of the papilla. They were not seen to anastomose with vessels derived from the central retinal artery. In about one-third of the dogs examined there was a well-marked physiological pit 1-2 dioptres deep ; but usually the papilla is flat with its periphery level with the inner surface of the retina, although its surface is often uneven compared with the papilla of a human eye. The papilla is usually pinkish white, but the colour may be very variable in the same dog on different occasions, and appears to be determined largely by changes in the smaller vessels near the periphery of the papilla, whose calibre closely reflects the general vasomotor tone, e.g. on a cold day with peripheral vasoconstriction the disc was paler than when the animal was in warm surroundings.

Observation of the choroid, other than the tapetum, is restricted to the dense brown pigment in its inner layers over the non-tapetal fundus. The pigment 
completely obscures the choroidal blood vessels in most normal dogs. In only one normal dog did we observe in the ventral non-tapetal fundus lighter beige-coloured streaks radiating out from the papilla and indicating the distribution of the main choroidal vessels. In very advanced cases of the hereditary generalized atrophy of Red Irish Setters when the atrophy of the choroid itself is advanced, one can occasionally observe these choroidal blood vessels radiating fanwise from the papilla.

(b) Histology.--The microscopic anatomy of the dog's retina follows the general mammalian pattern (Fig. 1). The structure and width of the layers

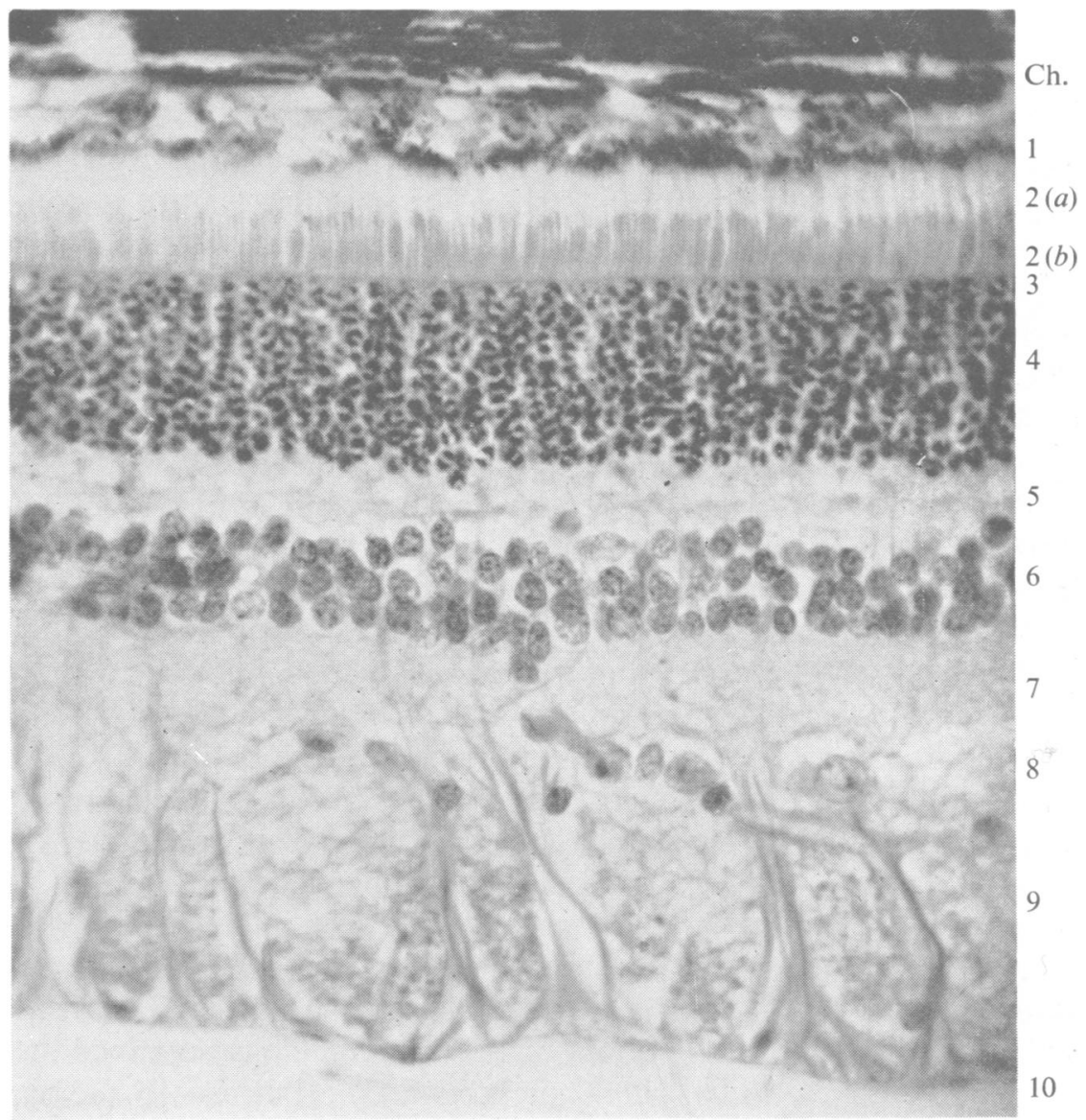

FIG. 1.-Section through retina of normal adult dog, non-tapetal fundus within $5 \mathrm{~mm}$. of the optic papilla. Note relative widths of layers, bundles of optic nerve fibres, and cones. Intra-vital fixation with Kolmer's cold-blooded fluid. Mallory's phosphotungstic acid haematoxylin. $(\times 350)$.

The following system of labelling the retinal structures, modified from Polyak (1941), applies to all the Figures:
Ch. Pigmented choroid.
Tap. Tapetum.
C.C. Chorio-capillaris.
E.Z. Eosinophilic zone.
Sc. Sclera.
1. Pigment Epithelium.
2. Layer of rods and cones:
(a) outer limbs; (b) inner limbs.
3. External limiting membrane.
4. Outer nuclear layer:
(a) cone nuclei; $(b)$ rod nuclei.
5. Outer fibre layer.
6 . Inner nuclear layer.
7. Inner fibre layer.
8. Ganglion cell layer. 
are similar over the whole fundus, except over the peripheral tenth, where the thickness of $200 \mu$ is reduced. There is no macula and the density of the cones remains remarkably constant throughout the central retina, about every fifth end organ being a cone as Schultze (1866) described. Rod-free portions of retina such as Zürn (1902) found have not been seen in normal eyes.

Bacillary (rod and cone) layer about $30 \mu$ wide with outer and inner limbs of approximately equal length (Fig. 2). The outer limbs stain yellow with the Orange $\mathrm{G}$ of the azan method, light blue with Mallory's phosphotungstic acid haematoxylin, dark blue with Heidenhain's method, and purple with Feulgen's method. The inner limbs stain deep blue with the azan method, and a

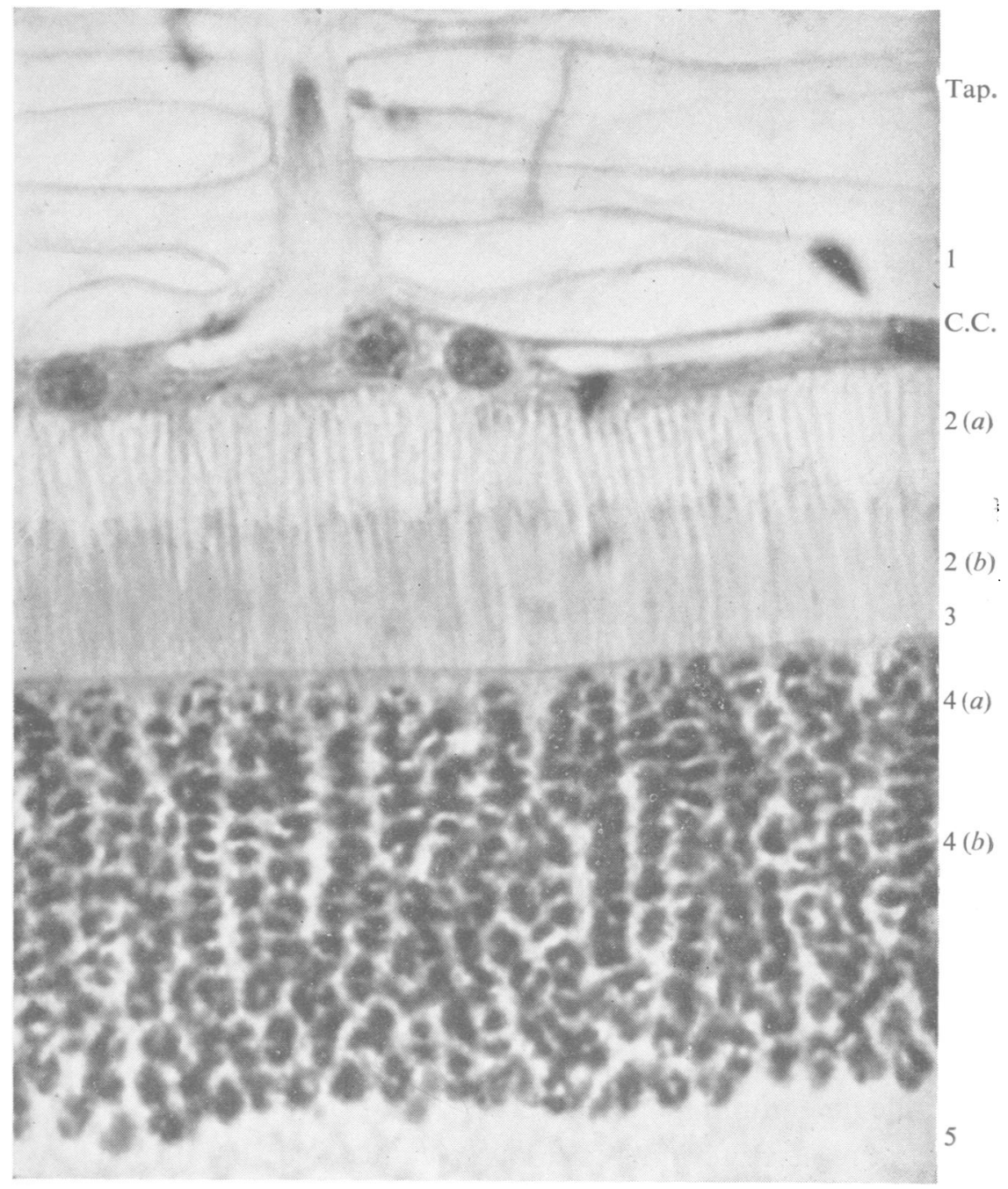

FIG. 2.-Section through outer part of retina of normal adult dog, mid-area centralis of the tapetal fundus. Note capillary traversing tapetum and branching to form chorio-capillaris. Pigment epithelium is free of pigment granules. Note pallisade arrangement of rod nuclei (4b). Intra-vital fixation with Kolmer's cold-blooded fluid. Mallory's phosphotungstic acid haematoxylin. $(\times 860)$. 
light bluish violet with Mallory's and Heidenhain's methods; they do not give a positive Feulgen reaction.

Outer nuclear layer about 70-80 $\mu$ wide and 10-12 nuclei thick, being reduced to $30-40 \mu$ and 5-6 nuclei over the peripheral non-tapetal fundus. The cone nuclei form a layer 1-2 nuclei thick immediately adjacent to the external limiting membrane ; they are about $15 \mu$ long and $10 \mu$ wide and have a more open chromatin network than that of the rod nuclei. The rod nuclei are smaller and rounder and their chromatin is denser and more lobulated. Both rod and cone nuclei stain a deep red with the azan method, deep blue with Weigert's haematoxylin, Heidenhain's haematoxylin, and Mallory's phosphotungstic acid haematoxylin; they give a well-marked positive Feulgen reaction (Fig. 2).

Outer fibre layer about $30 \mu$ wide, showing the three strata described by Polyak (1941) in primates, the outer stratum being the widest.

Inner nuclear layer (Fig. 3) about 35-40 $\mu$ wide and 3-4 nuclei thick (each 6-9 $\mu$ in diameter), being reduced to 2-3 nuclei thick in the peripheral fundus. Most of the nuclei are rounded with an open chromatin network, but along the outer margin are occasional larger nuclei surrounded by vacuolated cytoplasm, probably horizontal cells. The elongated or triangular nuclei of Müller's cells are inconspicuous. In our hands the nuclei of this layer stain rather differently from those of the outer nuclear layer, giving an indefinite Feulgen reaction and staining poorly with basic dyes and light brown with the azan method. In places adjacent to the larger blood vessels the layer was much reduced or even absent.

Inner fibre layer about $60 \mu$ wide without definite strata (Fig. 3).

Ganglion layer (Fig. 3) consisting of the giant, medium and small cells which were described by Ramon y Cajal (1894). The giant ganglion cells are about $30-35 \mu$ in diameter with a round nucleus $10-12 \mu$ in diameter, containing a dense nucleolus of $2-5 \mu$. The Nissl substance is well defined with as many as 30-40 granules in a plane of section. The medium ganglion cells are $15-25 \mu$ in diameter with nuclei similar in size to those of the giant cells. The small ganglion cells are $6-10 \mu$ in diameter and consist almost entirely of nucleus. The giant and medium cells are found spaced $20-200 \mu$ apart, being closer together near the papilla and less numerous towards the periphery, but their frequency varies very much in individual dogs irrespective of breed and age. The small cells are often two-deep and 10-20 $\mu$ apart in the peri-papillary zone but $50-100 \mu$ apart towards the periphery. The main retinal blood vessels lie in this and the nerve fibre layer ; they do not protrude above the general level of the inner limiting membrane.

Optic nerve fibres forming an inconspicuous layer $10-40 \mu$ thick over most of the retina, but in the zone $1 \mathrm{~mm}$. from the papilla rapidly increasing to $100 \mu$ (Fig. 1). These fibres are less dense than in other animals such as the sheep and are probably not myelinated. 


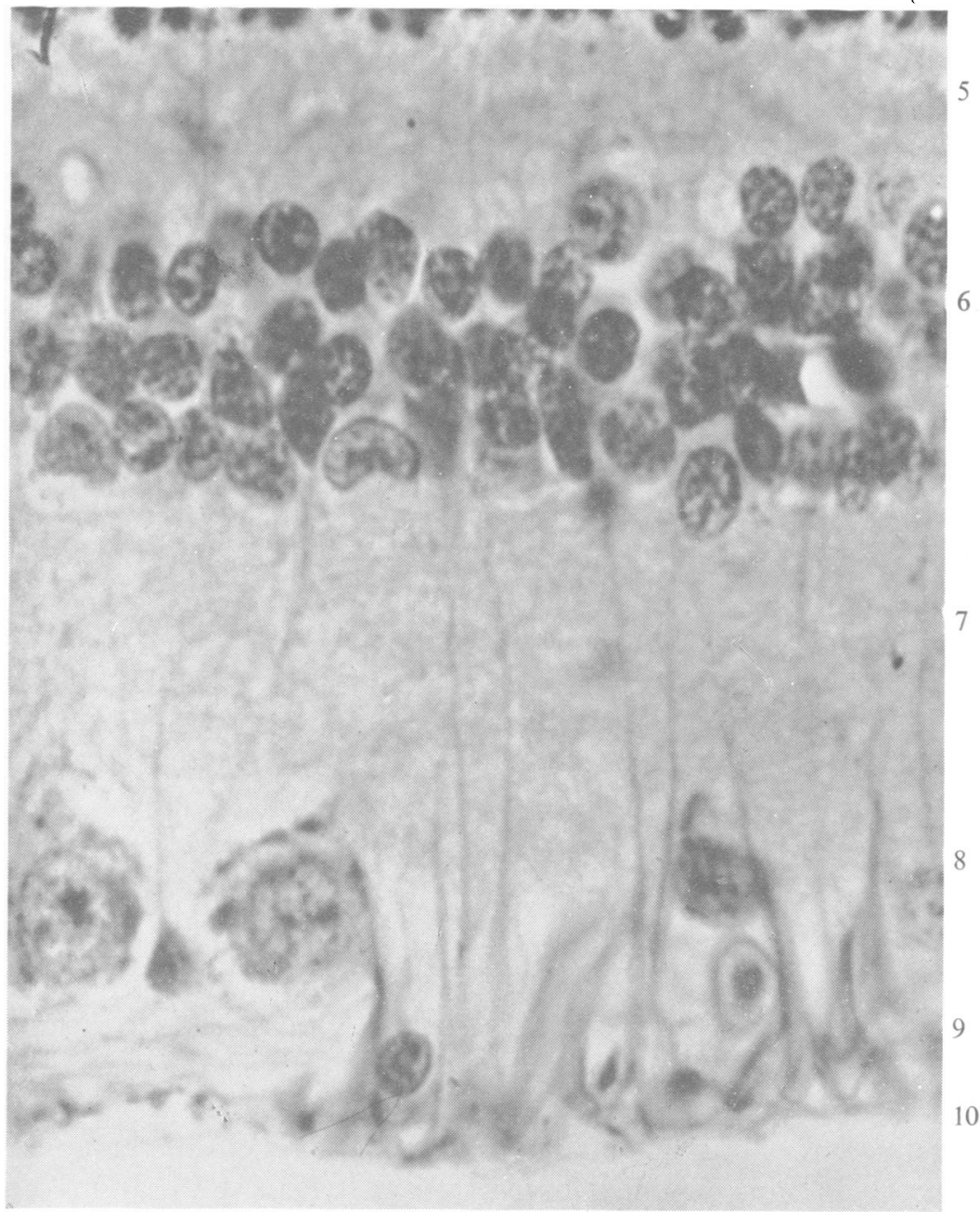

FIG. 3.- Section through inner part of same retina as that shown in Fig. 2. Note nuclei of inner nuclear layer which are larger than those of outer nuclear layer, Müller's fibres running through inner fibre layer (7) and the giant, medium and small ganglion cells $(8)$. $(\times 860)$.

Internal limiting membrane very fine.

Cells of the pigment epithelium about $10 \mu$ wide; their nuclei have dense chromatin staining a deep red with the azan method and occupy the full width of the cell. The pigmentation varies and can be described in four zones: tapetal, junctional, non-tapetal, and peripheral. In the tapetal zone there are no visible organized pigment granules (Fig. 2). In the junctional zone at the periphery of the tapetum, a single layer of small lanceolate granules appears at the inner margin of the cells or in their processes. In the nontapetal zone (Fig. 1), lanceolate pigment granules form a dense mass of radially arranged particles in the inner third of each cell and extend $1-2 \mu$ into the 
processes, the outer portion of the cells remaining firee of pigment. In the peripheral zone over the extreme peripheral $2-3 \mathrm{~mm}$. of the fundus, the cell cytoplasm is filled with round granules of dense brown pigment often $1-2 \mu$ in diameter similar to those of the choroid.

Tapetum lucidum cellular, consisting of a layer up to eight cells thick of elongated rectangular cells with parallel sides set at right angles to the optic axis (Fig. 2). These cells have a small dense nucleus and scanty cytoplasm free from pigment. The tapetum thins towards its periphery and has never been observed to extend to the peripheral margins of the retina. Capillaries connecting the chorio-capillaris to the choroidal blood vessels traverse the tapetum at an angle of $15-30^{\circ}$ to the perpendicular radius, forming the stellulae of Winslow.

Choroid (Fig. 1) about 150-300 $\mu$ wide, consisting of elongated cells similar to those of the tapetum but densely loaded with dark brown pigment granules which are very small $(0 \cdot 5 \mu$ diameter $)$.

(c) Occasional Anomalies.-We have observed colobomata adjacent to the optic papilla as described by Nicholls and Tansley (1938), and Blessig-Henlé cavities in the extreme peripheral $500 \mu$ of the non-tapetal fundus as described by Zürn (1902). The incidence of each anomaly was about 1.5 per cent of the eyes examined histologically.

(2) Development of the Retina in the Young Puppy.

(a) Clinical Data.-The young puppy does not appear to see well until about six weeks after birth, i.e. about 100-105 days post-conception, when it has usually been weaned. Until this time the media are not clear, it is not possible to carry out night vision tests satisfactorily, the electro-retinogram is not fully developed (Parry, Tansley and Thomson, 1951), the vitreous humour is a white opaque mass when the eyeball is opened after fixation and the layers of the retina have not differentiated fully. The state of differentiation varies considerably in pups of similar post-natal age, but these variations are much reduced when the post-conception age is taken into account. The gestation periods of the pups used in these studies varied from 58 to 68 days, the majority being 60 to 63 days. The post-conception age was found to be a more reliable guide to a pup's physiological maturity than the post-natal age.

Puppies are born with unopened eye-lids; the lids open about the 72nd $t$ 2 days post-conception (10-15 days after birth). The media are not transparent at this time and it is not until 95-105 days post-conception (5-6 weeks after birth) that they are as clear as in the adult eye. From about the 78th-80th days post-conception (18-20 days after birth) pupillary light reactions are detectable and the fine blood vessels of the retina appear to be partly buried. Occasionally the remnants of the hyaline vessels are observed up to 3 weeks after birth. The tapetum looks flat, grey, and of a fine granular texture, but its detailed structure could not be observed owing to difficulty in focusing sharply. From the 90th day post-conception (about 4 weeks after birth) the tapetal reflection is deep violet-grey and the 
stellulae of Winslow can be seen. The main blood vessels are well marked and the fine branches over the retina sharply defined. The papilla is well demarcated. During the following weeks the colour of the tapetal reflection changes through a bluish dove-grey to the adult colour, which is seen usually 8 weeks after birth, when the normal adult texture is also present.

(b) Histology.-The retina of the newborn pup is very much less well developed than the retina of the newborn child. The stage of development in the newborn pup corresponds roughly to the early third stage (Mann, 1928) seen in the human embryo of the third or fourth month (Mann, 1928 ; Duke-Elder, 1932), and the developments of the late third and fourth stages occurring in man during the fourth to eighth months of pregnancy take place in the dog's retina during the first 6 weeks after birth, i.e. between the 60th and 105th day post-conception.

At the 60 th day post-conception there is no rod and cone layer and no outer fibre layer, and the inner and outer nuclear layers form a single combined nuclear layer comprising the undifferentiated outer neuroblastic layer and presumably those cells of the inner neuroblastic layer which will form Müller's cells and the amacrine cells of the inner nuclear layer. This combined nuclear layer is $80-120 \mu$ wide and 30 nuclei thick ; the nuclei are all similar being

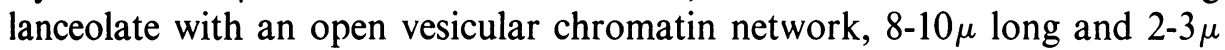
wide and over-lapping each other without any regular pallisade arrangement. The techniques employed gave no indication as to which cells would eventually form the outer and inner layers, but Tansley (1933a) has demonstrated such a difference in the rat retina at a similar stage of development by using osmic acid fixation. At the outer margin of the combined nuclear layer and separating it from the external limiting membrane lies an eosinophilic zone of hyaline tissue. In this zone, $50-100 \mu$ apart, are nuclei $10-12 \mu$ in diameter in the various stages of mitosis. On the outer surface of the external limiting membrane are the primordia of the rods and cones $0.5 \mu$ long and contiguous with the pigment epithelium, the cells of which contain little or no pigment except in the peripheral zone. The inner fibre layer is about $7 \mu$ thick. In the ganglion layer the cells are often two deep, most being about $10 \mu$ in diameter with a few cells $20 \mu$ in diameter near the papilla. The optic nerve fibres are inconspicuous and the differentiation of the tapetum from the choroid is indistinct with the choroid only containing traces of pigment in its inner cells.

About the 62 nd day post-conception cleavage of the combined nuclear layer begins over the area centralis and is complete about the 66th to 68th day. The separation usually leaves an outer nuclear layer of 15-18 nuclei and an inner one of 9-10 nuclei, but sometimes the relative widths are reversed. The nuclei are still largely undifferentiated, but cell division has ceased, some cone nuclei can be recognized and some of the nuclei along the margins of the inner nuclear layer are becoming rounded. The large ganglion cells are bigger, more evenly distributed over the fundus and show some Nissl granules. The retinal blood vessels are much larger and are raised above the inner surface of 
the retina. The rods and cones are still very immature, only $2-2 \cdot 5 \mu$ long and jutting outwards through the external limiting membrane. The tapetum is a distinct layer of oval cells with fairly dense cytoplasm and the choroid contains more pigment. The adult nuclear staining reactions have not yet appeared, the nuclei of both inner and outer layers staining a uniform brownish-red with the azan method and red-purple with Feulgen's method.

About the 80th day post-conception the adult nuclear staining reactions are present, and the outer and inner limbs of the rods and cones are quite distinct, being $4-5 \mu$ and $3-4 \mu$ wide respectively. However there is still an eosinophilic zone between the external limiting membrane and the cone nuclei which do not yet form a continuous layer. Occasional pyknotic rod nuclei are present in every 4-6 fields (with 1/6" objective), i.e. 1 in 5-10,000 nuclei. In the inner nuclear layer most nuclei have differentiated and the nuclei and processes of Müller's cells are conspicuous for the first time. The optic nerve fibres are

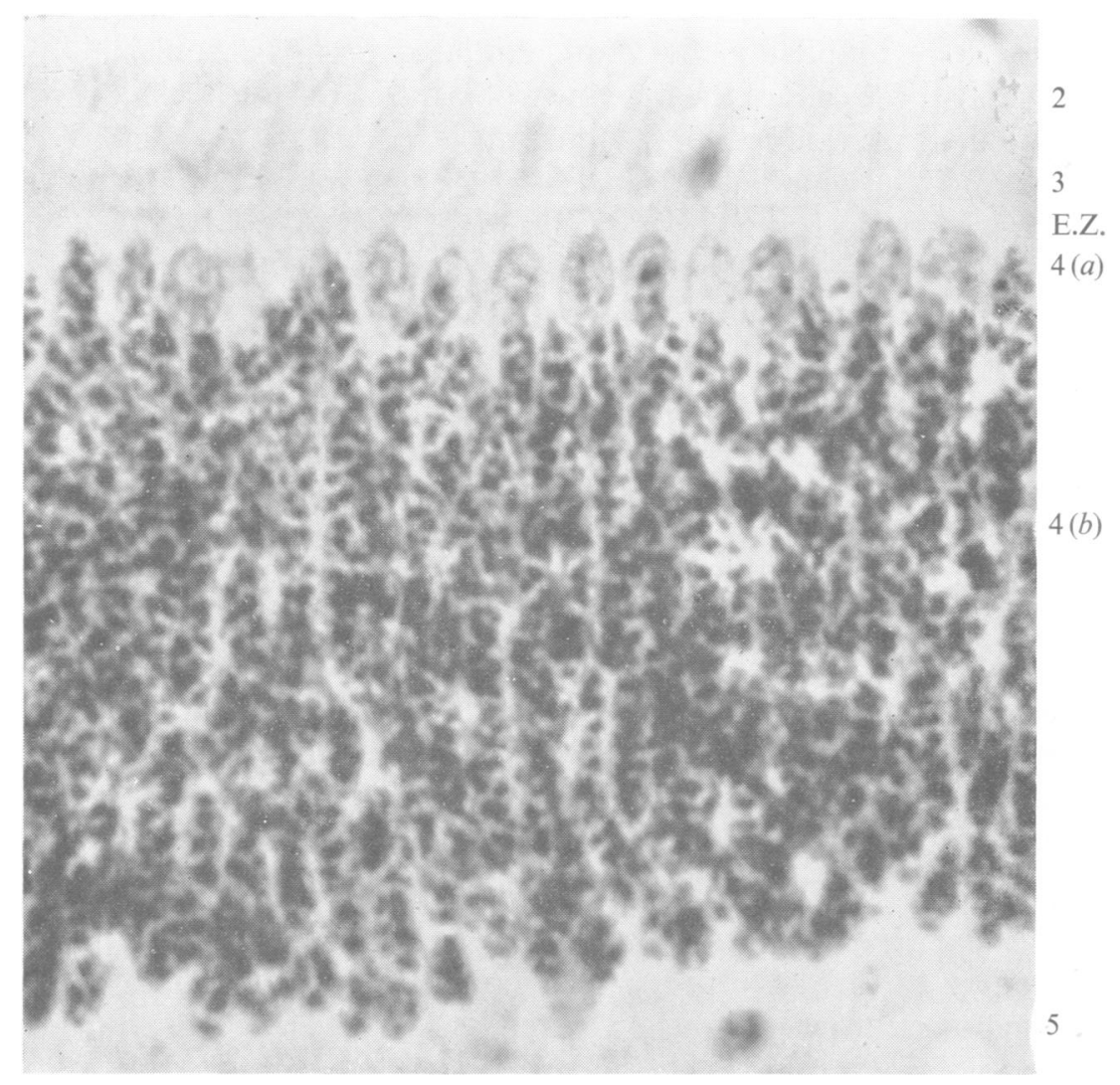

FIG. 4.- Section through retina of normal pup aged 92 days post-conception, mid-area centralis of tapetal fundus. Note great development of rods and cones (2). Nuclei of cones are quite distinct from those of rods whose chromatin is more lobulated. Cone nuclei (4a) now form a nearly contiguous layer immediately adjacent to outer limiting membrane, and eosinophilic zone has almost disappeared. Intra-vital fixation with Kolmer's cold-blooded fluid. Mallory's phosphotungstic acid haematoxylin. $(\times 860)$. 
distinct and the Nissl substance of the larger ganglion cells is beginning to disintegrate into discrete granules.

This stage of development corresponds very closely with our earliest detection of the electroretinogram (Parry, Tansley and Thomson, 1951). We were unable to detect any potentials in pups at the 80th day post-conception, but at the 83 rd day a small $b$-wave was recorded, which became larger until the 100-105th day when the normal adult form of ERG was obtained.

Between the 85th and 95 th days post-conception the rods and cones acquire their adult size and staining reactions. The eosinophilic zone disappears, presumably due to extrusion of its substance through the external limiting membrane to form the rods and cones. The cone nuclei are now close to the external limiting membrane as a clear-cut layer one nucleus thick with the nuclei spaced at first 2-2.5 nuclear diameters apart but becoming contiguous (Fig. 4). The rod nuclei are becoming more oval and their chromatin lobulated. The nuclei of the inner nuclear layer are similar to those of the adult retina and in the large ganglion cells the Nissl substance is now distinct. The blood vessels have now penetrated the inner retina and numerous capillaries can be seen traversing the inner nuclear layer and forming a network in the outer fibre layer. The chorio-capillaris is well developed and the tapetal cells are elongated.

By the 100-105th day post-conception the retina and tapetum appear as in the adult, while the pigmentation of the pigment epithelium and choroid is also complete.

The retina once mature shows little change up to the age of 6 years ; we have not had suitable material older than this for study.

\section{(3) Changes in the Retina when Fixed after Death}

We examined eyes from some forty normal dogs fixed at various periods up to $48 \mathrm{hrs}$ after death, including some fixed intravitally immediately after death. The usual fixatives were used. The carcases were kept in the shade at room temperature, i.e. between $40^{\circ}-70^{\circ} \mathrm{F}$., and in a few instances the sequence of changes seen in the eyes of different dogs was confirmed as occurring in the same animal by removing one eye and allowing the second eye to remain in the carcase for a longer period.

The changes in dogs more than 8 weeks old are remarkably uniform, and may be conveniently described in four stages.

Stage 1 (Fig. 5, overleaf) is seen when fixation takes place within 30 minutes of death; the earliest signs can be detected in most preparations fixed within five minutes after death without intravascular injection of fixative. The outer limbs lose their dense palisade arrangement and a space appears between each group of 3 to 5 processes. At the same time the beading of the segment becomes more obvious. In the inner and outer nuclear layers, about $0 \cdot 1$ per cent. of the nuclei show pyknosis of the chromatin, which is rounded, very dense, and stains a very deep blue-black with haematoxylin, a deep red with 


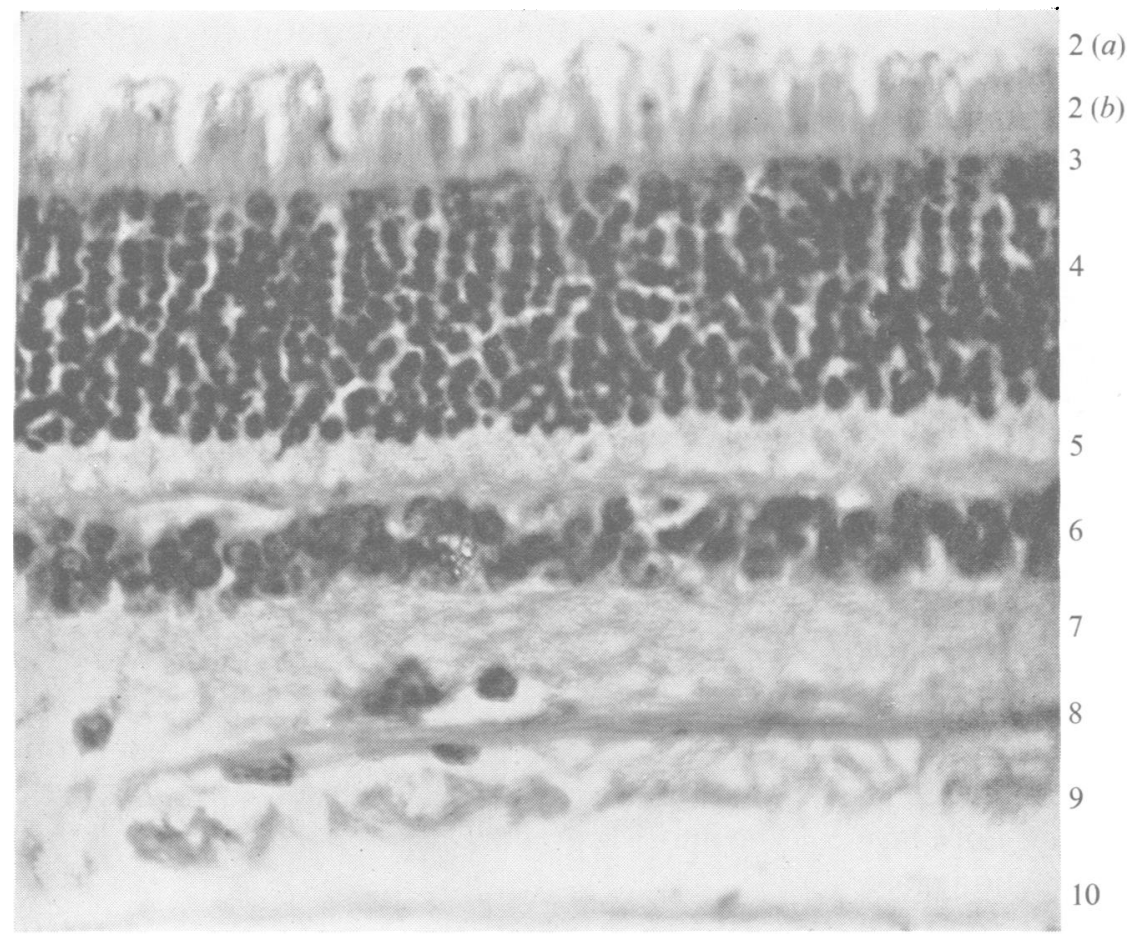

Fig. 5.- Section through retina of normal adult dog, fixed 30 minutes after death, mid-ventral non-tapetal fundus. Note fragmentation of outer segments of rods and their separation from pigment epithelium. Chromatin of nuclei is still largely unaffected, but there is a general loss of fine definition. Kolmer's cold-blooded fluid. Mallory's phosphotungstic haematoxylin acid. $(\times 440)$.

azo-carmine, and purple with Feulgen's method. The remainder of the retina is normal.

Stage 2 (Fig. 6) develops between 1 and approximately 6 hrs after death. The outer limbs, especially at points adjacent to the pigment epithelium, become broken up into their constituent discs, thereby causing detachment of the retina from the pigment epithelial layer and making these sections very difficult to prepare without separation of the retina. The inner limbs are essentially normal and the inner remnants of the outer limbs still give a positive Feulgen reaction. The pyknosis of the rod nuclei increases progressively and by 1-2 hrs after death nearly half are contracted with pyknosis of the chromatin, but there is no contraction of the mass of the outer nuclear layer, so that the rod nuclei individually appear further apart. The cone nuclei are still unaffected.

In the inner nuclear layer up to 10 per cent. of the nuclei show pyknosis, with contraction of the nuclei to about half the normal diameter. At first these pyknotic nuclei are more numerous along the inner margin of the layer and the nuclei of Müller's cells appear to be affected preferentially, but gradually the affected nuclei appear evenly throughout the layer. The staining reactions of these pyknotic nuclei differ very much from those of the normal 


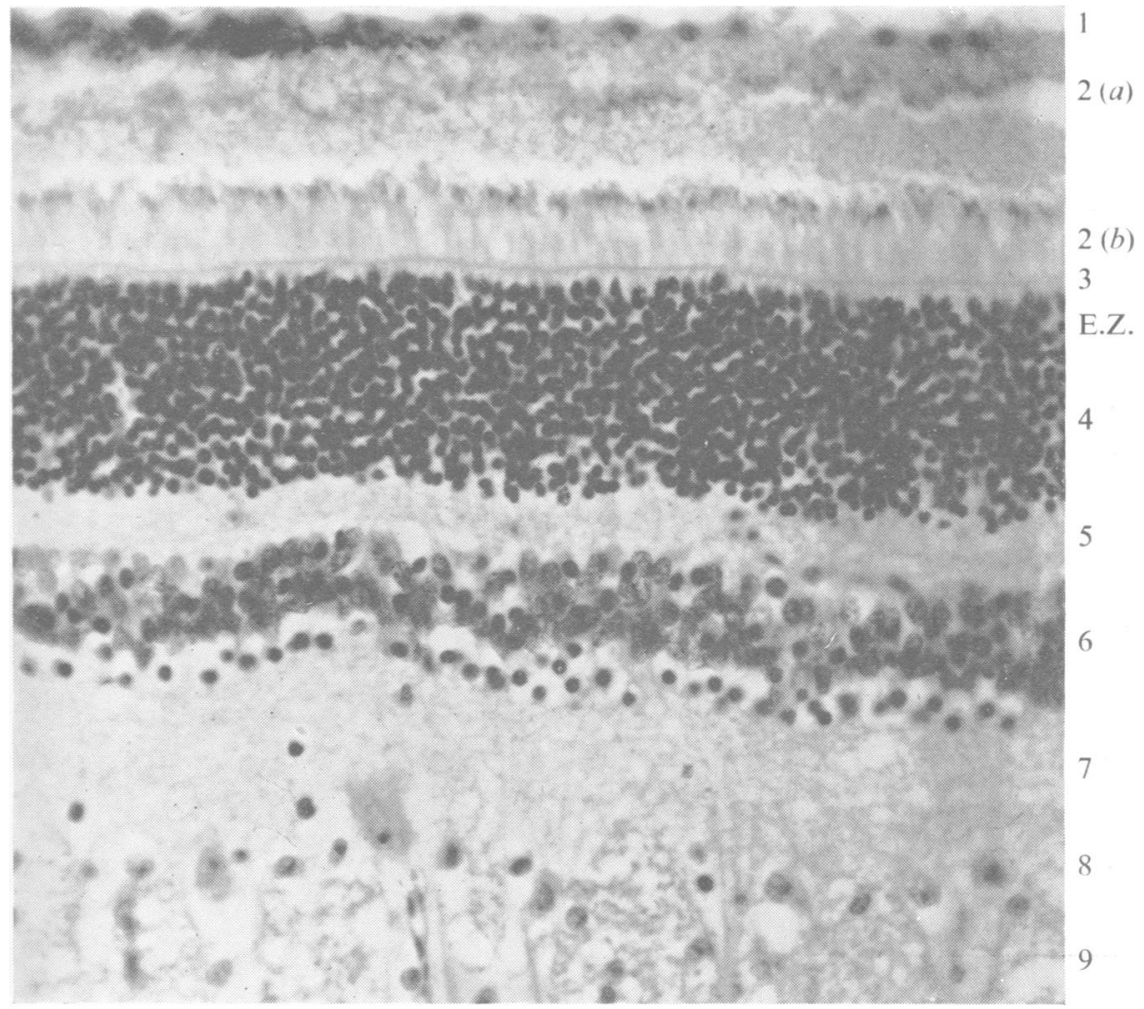

FIG. 6.-Section through retina of normal adult dog fixed 6 hrs after death, peri-papillary non-tapetal fundus. Note disintegration of outer limbs of rods $(2 a)$, while inner limbs $(2 b)$ still retain much of their normal structure. The cone nuclei are retracted from outer limiting membrane, leaving a clear eosinophilic zone at outer margin of outer nuclear layer. Most rod nuclei (4) are pyknotic, as are some nuclei of the inner nuclear layer (6). Optic nerve fibres are becoming fragmented. Kolmer's cold-blooded fluid. Mallory's phosphotungstic acid haematoxylin. $(\times 360)$.

nuclei with their open reticular chromatin staining a very light blue-purple with haematoxylin, blue rather than purple with Feulgen's method, and a light chestnut-brown with the azan method. The pyknotic nuclei stand out as dense round masses of chromatin sharply demarcated, staining a deep purple with haematoxylin, a deep red with azo-carmine, and a deep purple with Feulgen's method. The ganglion cells show some loss of Nissl substance which is less granular, and the nucleoli stain a deep red with azo-carmine. The fibre layers and limiting membranes are still essentially normal, although the internal limiting membrane begins to separate from the optic nerve fibres.

Stage 3 (Fig. 7, overleaf) is seen in eyes fixed 6 to $18 \mathrm{hrs}$ after death. The most conspicuous sign is a regular folding of the retina which appears in sections as folds and rosettes. The thickness of the portion of the retina between the outer and inner limiting membranes remains constant over much of the fundus, but it is about 10 per cent. greater than normal at $250-300 \mu$. This increase is due largely to the greater width of the outer nuclear layer which is 


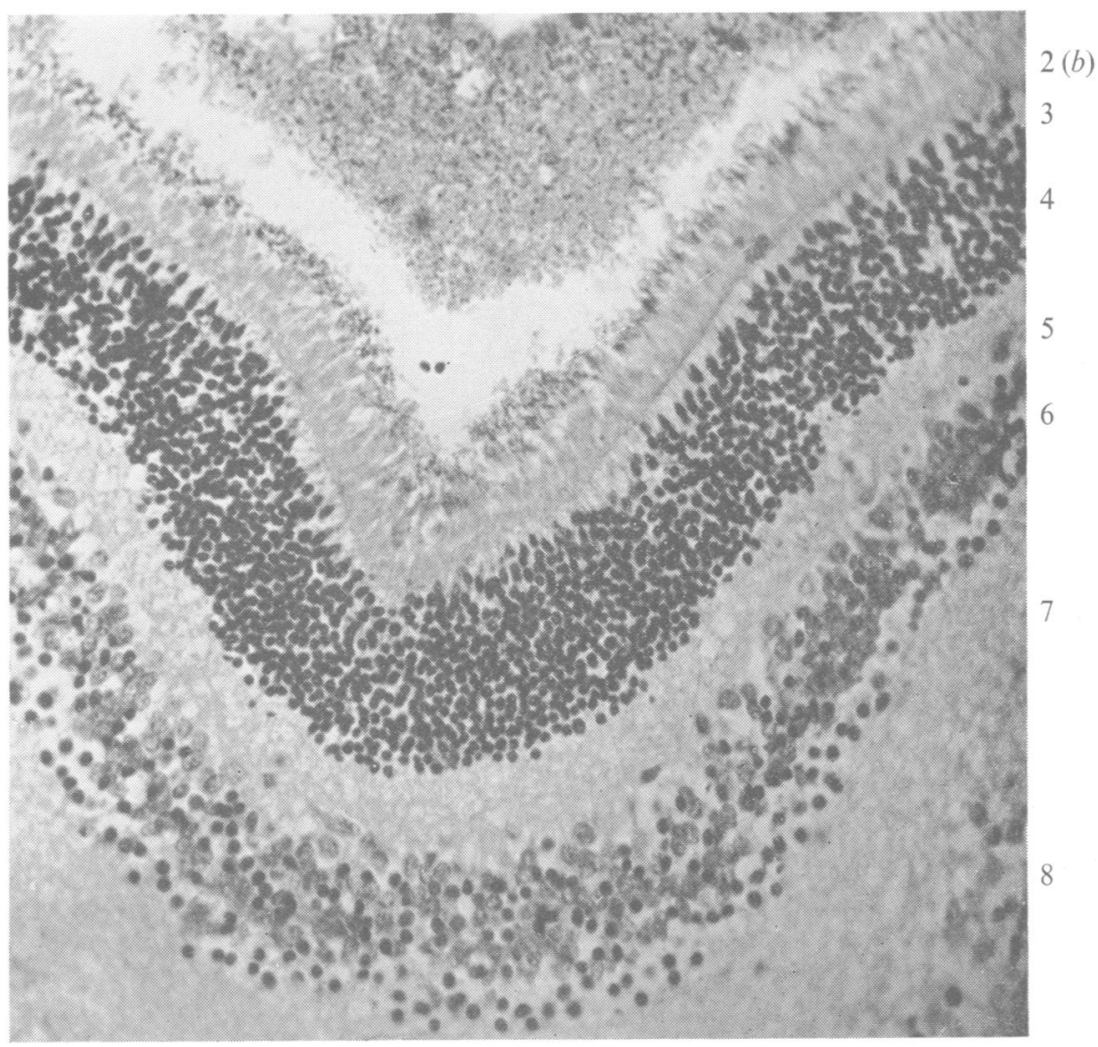

Fig. 7.- Section through retina of second eye of same dog as that shown in Fig. 6, but not fixed until $18 \mathrm{hrs}$ after death. Note folding of retina due to swelling and separation, chiefly of outer limbs of rods. Nuclei of outer nuclear layer (4) are all pyknotic, as are many nuclei of inner nuclear layer (6). General fragmentation of fibre layers. Kolmer's cold-blooded fluid. Mallory's phosphotungstic acid haematoxylin. $(\times 320)$.

about $100-120 \mu$ (normal $70-80 \mu)$. The variations in total thickness are due to disintegration of the rods and cones, which become progressively fragmented and the remnants swollen. At first this fragmentation remains confined to the outer $3 / 4$ of the outer limbs and even at $8 \mathrm{hrs}$ post mortem the inner limbs are relatively intact and the attached remnants of the outer limbs still give a positive Feulgen's reaction. Thereafter this is lost and the inner limbs fragment. The external limiting membrane is at first intact but the outer nuclei of the outer nuclear layer become separated from the membrane by a zone of eosinophilic tissue, as in very young pups. The membrane has usually disintegrated completely by $12 \mathrm{hrs}$ after death. Meanwhile the nuclei of the outer nuclear layer become separated further from each other until they are about the diameter of one pyknotic nucleus apart $(8-10 \mu)$. The pyknosis of the nuclei of the outer nuclear layer increases gradually until half and later all are affected, the cone nuclei being more resistant, while in the inner nuclear layer pyknotic nuclei increase until about half are so affected. No fine cytoplasmic structure can be seen in the ganglion cells although their 
processes are conspicuous, while the chromatin of the nuclei of the small ganglion and glial cells is rounded and stains very deeply. The optic nerve fibres begin to swell and fragment and there is considerable loss of substance in the fibre layers. The internal limiting membrane is swollen and disintegrating as are Müller's fibres. The cells of the pigment epithelium and their pigment begin to disintegrate late in this stage after the cytoplasm of the tapetal cells has swollen.

Stage 4 is seen in material fixed more than $18 \mathrm{hrs}$ after death. Nearly all the nuclei are pyknotic, and the fibre and bacillary layers have disappeared, leaving only a mass of less densely packed pyknotic nuclei at the site of the nuclear layers suspended in a very faintly staining eosinophilic matrix. Nuclear counts have been possible on material fixed up to $48 \mathrm{hrs}$ after death, but thereafter the recognition of the structural features becomes more and more difficult as the whole retina disintegrates.

In animals less than 8 weeks old the pyknosis of rod nuclei occurs less rapidly and Stage 2 does not develop unless fixation is delayed until some 4 hrs after death. In these eyes, fragmentation of the outer limbs is the main sign of early post-mortem change.

\section{Discussion}

The gross structure of the retina of the dog is similar to that usually seen in carnivores (Schultze, 1866 ; Ramon y Cajal, 1894 ; Zürn, 1902 ; Walls, 1942), but there are certain details which call for further comment.

A rod-free fovea externa with an increased density of cones and their nuclei has been described by Zürn (1902) in certain breeds which hunt by sight rather than by scent, e.g. German gun dogs, Russian greyhounds etc., but not in others such as certain terriers which hunt by scent. We examined particularly racing greyhounds as an example of a dog hunting by sight, while the setters and retrievers are good examples of dogs hunting mainly by scent. We were unable to confirm Zürn's observations of a rod-free fovea, nor did we detect any area of increased cone density. In fact, the regularity of the cone density, as determined in radial sections of the normal retina and in the density of the residual cone nuclei seen in the rod-free retinae of dogs suffering from hereditary degeneration of the retina (Parry, 1953), is very striking. Our observations support the contention of Walls (1942) that animals with a tapetum do not have a fovea, the advantages of increased visual acuity provided by a fovea being nullified by the tapetal reflection. In addition, the density of the ganglion cells is also remarkably constant.

Compared with many other domestic animals and with man, the dog has a large number of rod nuclei. If we assume that each cone is connected to a single cone bipolar cell, as studies of the dog retina by Ramon y Cajal (1894) suggest, then each rod bipolar is connected to at least 8-10 rods. The recognition of the nuclei of amacrine, horizontal, and bipolar cells in the inner nuclear layer is not easy by the methods described in this paper, but the nuclei of Müller's cells can easily be distinguished. 
The ganglion layer has cells of two types, the large with abundant cytoplasm and the small with very little cytoplasm. The large cells are the giant and medium. ganglion cells with their conspicuous Nissl's granules; their density is rather higher in the peri-papillary fundus than elsewhere. The small cells are small ganglion cells. Whether in the dog retina the cone and rod bipolars are specially connected to any of the three kinds of ganglion cells is not known, but there would appear to be a considerable concentration of both rod and cone connections to each ganglion cell, the bipolar cells being 15-20 times as numerous as the ganglion cells.

The surface of the optic papilla is nearly always on the same level as the surrounding retina, as Wolff and Davis (1931) reported ; in some 20 per cent. of eyes only is there a well marked physiological pit, less extensive than the cup in man.

The differentiation of the dog's retina occurs at a very much later stage of general development than in man. Thus in man (Mann, 1928 ; Duke-Elder, 1932), cell division is not seen after the $11-\mathrm{mm}$. stage, when Müller's fibres are already present ; the differentiation of nuclei of the potential inner and outer nuclear layers can be seen at the $48-\mathrm{mm}$. stage, and the outer fibre layer is present at the $110-\mathrm{mm}$. stage (4th month of pregnancy), when small rods and cones are formed; all these stages occur in the dog's retina after birth. However the maturation of the dog's retina after birth follows the sequence described by Tansley in the rat (1933b) and in the mouse (1951), although proceeding over a longer period. The relatively late development of the cones and rods about the 75-80th day post-conception after the differentiation of the two nuclear layers is well advanced is of interest, since it coincides so closely with the earliest detection of an electroretinogram and with the development of the staining reactions of the mature retina. The cones appear a few days before the rods, whose nuclei also mature more slowly. The limb segments of the rods and cones continue to elongate until about the 100-105th day post-conception ( 6 weeks after birth), being the last portion of the retina to assume a mature form. The cone nuclei are probably scattered through the outer portion of the primitive nuclear layer and actively migrate until they lie as a solid layer of contiguous nuclei against the external limiting membrane. It is difficult to explain the gradual completion of this layer in a constantly enlarging eyeball without such active migration. The number and distribution of the ganglion cells also suggest a similar migration, as well as post-natal development from primordial cells ; in the newborn pup the large cells of the ganglion layer are few and far between except for a narrow zone around the optic papilla, where they are very much more numerous than in the adult, and small cells are distributed evenly throughout the retina in numbers considerably greater than in the adult. It seems probable, therefore, that the ganglion cells of the mature retina are derived partly from a centrifugal migration of the peri-papillary large ganglion cells present at birth, and partly from post-natal differentiation from the primordial cells of the 
ganglion layer. It is of interest that the nerve fibre layer becomes much more distinct between the 70th and 80th day post-conception.

The retina of the newborn pup is essentially avascular. The circulation from the central retinal vessels is confined to a fine network near the inner surface, embedded in the rudimentary nerve fibre layer, and it is not until three weeks after birth that the adult arrangement with capillaries extending to the outer fibre layer is seen. At the same time the surface vessels become larger with their inner surface at the surface of the retina, a fact which explains the ophthalmoscopic observation that the retinal vessels appear to be buried at first but to reach the surface as distinct vessels about the 90th day postconception. The retinal blood vessels arise from two sources, the central retinal artery and from subsidiary arterioles arising directly from choroidal vessels at the periphery of the papilla. We have not traced in detail the origin of the central retinal artery, but recently it has been suggested that it arises from the ciliary artery (Moffat, 1952).

The gradual reduction of the width of the nuclear layers during infancy and adolescence can be explained satisfactorily by the gradual enlargement of the eyeball. There may be a very slow reduction of the nuclei during middle age and senescence, but the number of dogs in this group, with unimpeccable medical histories with regard to virus infections is insufficient to form a reliable estimate.

The changes in the retina occurring after death and producing artefacts to which Birch-Hirschfeld (1900) drew attention in human material, are remarkably constant and specific in the dog in the sense that the nuclear changes are quite distinct from any we have seen in properly fixed dystrophic retinae and it is possible to distinguish these post-mortem changes from dystrophic changes in many abnormal eyes. The folding and rosette formation could be explained readily by assuming that the swelling, which we know occurs over the width of the retina also occurs transversely, i.e. the postmortem changes cause a general increase in the volume of the retina, so that it tends to fold and in sections to produce rosette formations. The pigment epithelium shows no signs of hypertrophy or of pigmentation of the cytoplasm in the body of the cell.

\section{Summary}

(1) The results of clinical and ophthalmoscopic examinations of the eye are correlated with the histology of the retina in a series of normal dogs of various breeds.

(2) No striking differences were noted in dogs hunting by sight and by scent, and no evidence of an area of retina with cones only was found.

(3) The rod and cone nuclei are some 8 to 10 times as numerous as the bipolar cells, which are themselves 15 to 20 times as numerous as the ganglion cells, which are of three sizes. 
(4) The development of the retina from birth, when the nuclear layers have not differentiated, to the adult form at about six weeks after birth (100-105th day post-conception) is compared with that in man.

(5) The changes in the retina occurring when fixation has been delayed for various periods of up to $48 \mathrm{hrs}$ after death are described.

I should like to thank Dr. Katharine Tansley, who has made freely available the results of her wide experience of retinal histology and has kindly read the manuscript. I am particularly indebted to Mr. C. F. Hart and his assistants for making the histological preparations.

\section{REFERENCES}

Andersen, A. C., and Hart, G. H. (1943). Amer. J. vet. Res., 4, 307.

BiRCH-HiRSCHFELD, A. (1900). v. Graefes Arch. Ophthal., 50, 166.

DUKe-Elder, S. (1932). "Text-book of Ophthalmology," vol. 1, p. 326. Kimpton, London.

EINARSON, L. (1951). Acta path. microbiol. scand., 28, 82.

Hart, G. H., and Guilbert, H. R. (1937). J. Amer. vet. med. Ass., 91 (44 new series), 193.

Hodgman, S. F. J., Parry, H. B., Rasbridge, W. J., and Steel, J. D. (1949). Vet. Rec., 61, 185. Johnson, G. L. (1901). Philos. Trans., B, 194, 1.

JoHNSON, M. L. (1939). J. exp. Zool., 81, 67.

(1943). Arch. Ophthal., Chicago, 29, 793.

ManN, I. C. (1928). "The Development of the Human Eye." University Press, Cambridge.

MANSI, W. (1951). Brit. vet. J., 107, 214.

Mellanby, E. (1943). J. Physiol., 101, 408.

MOFFat, D. B. (1952). Nature, Lond., 169, 1015.

Moore, L. A., Huffman, C. F., and Duncan, C. W. (1935). J. Nutr., 9, 533.

Nicholls, J. V. V., and TANSLEY, K. (1938). British Journal of Ophthalmology, 22, 165.

NiCOLAS, E. (1925). " "Veterinary and Comparative Ophthalmology". (Trans. and enlarged by H. Gray). Brown, London.

OAKLeY, C. L. (1937). J. Path. Bact., 44, 365.

PARrY, H. B. (1951). Vet. Rec., 63, 323.

(1953). British Journal of Ophthalmology, 37. In the press.

- - and LarIn, N. M. (1951). Vet. Rec., 63, 833.

—_, TANSley, K., and Thomson, L. C. (1951). J. Physiol., 115, 47 P.

POC (1953). Ibid., 120, 28.

PolyaK, S. L. (1941). "The Retina ". Chicago University Press.

RAMON y CAJAL, S. (1894). "Die Retina der Wirbelthiere." (Trans. and ed. by R. Greeff.)

Bergmann, Wiesbaden.

SAUNDERS, L. Z. (1952). Cornell Vet., 42, 67.

SCHULTZE, M. (1866). Arch. mik. Anat., 2, 175.

TANSLEY, K. (1933a). British Journal of Ophthalmology, 17, 321.

- (1933b). Proc. roy. Soc., Lond., B., 114, 79.

(1951). British Journal of Ophthalmology, 35, 573.

WALls, G. L. (1938). Stain Technol., 13, 69, and personal communication.

(1942). "The Vertebrate Eye and its Adaptive Radiation." Cranbrook Institute of

Science, Michigan.

WoLfF, E., and DAVIES, F. (1931). British Journal of Ophthalmology, 15, 609.

Zürn, J. (1902). Arch. Anat. Physiol., Lp. 3., Anatom. Abt. Supplement-band, p. 99. 